\title{
How nescience may obscure evidence
}

\author{
M.-L. Sautter-Bihl · F. Sedlmayer · W. Budach $\cdot$ J. Dunst $\cdot$ P. Feyer $\cdot$ R. Fietkau $\cdot$ W. Haase $\cdot$ W. Harms $\cdot$ \\ M.D. Piroth - R. Souchon - F. Wenz $\cdot$ R. Sauer $\cdot$ Breast Cancer Expert Panel of the German Society of Radiation \\ Oncology (DEGRO)
}

Published online: 20 September 2014

(C) Springer-Verlag Berlin Heidelberg 2014

During the last decade, treatment trends for early breast cancer have been fluctuating between opposite extremes. More aggressive regional nodal irradiation (RNI) has been suggested by several recent studies [10,17], on the other side, the previously unquestioned dogma of axillary dissection as an important part of breast cancer management was gradually abandoned in favor of sentinel node dissection (SLND). While consensus was rapidly achieved for pathologically negative $\mathrm{SN}$, the management of patients with one or two positive SN remained controversial up to the first publication of a randomized study of the American College of Surgeons Oncology Group [3] which specifically addressed the outcome of such patients with ALND vs. none. Even though

Editorial on Jagsi et al. Radiation field design in the ACOSOG Z0011 (Alliance) Trial.

Prof. Dr. med. M.-L. Sautter-Bihl ( $₫)$

Klinik für Radioonkologie und Strahlentherapie, Städtisches

Klinikum Karlsruhe gGmbH,

76133 Karlsruhe, Moltkestr. 90, Germany

e-mail: marie-luise.sautter-bihl@klinikum-karlsruhe.de

F. Sedlmayer

Department of Radiotherapy and Radiation Oncology, LKH

Salzburg, Paracelsus Medical University Hospital,

Salzburg, Austria

W. Budach

University Hospital Düsseldorf,

Düsseldorf, Germany

\section{J. Dunst}

University Hospital Schleswig-Holstein,

Lübeck, Germany

P. Feyer

Klinikum Neukölln,

Berlin, Germany limitations of the study were recognized and let to some critical comments [7], current guidelines [8, 14] rapidly adopted the omission of ALND in these selected patients.

Unreckoned insights about the quality of evidence generated by the Z0011-trial were provided by a recent publication on behalf of the Alliance for Clinical Trials in Oncology by Jagsi et al. Briefly, the study [3] (published in 2011) comprised 891 women from 115 institutions with a clinically negative axilla who underwent sentinel node dissection (SLND), revealing 1-2 pathologically affected nodes. Tumor characteristics were pT1 $(70 \%)$ or pT2 invasive carcinomas, mostly ER +, well- differentiated tumors. Patients were randomized to either axillary dissection (ALND) or

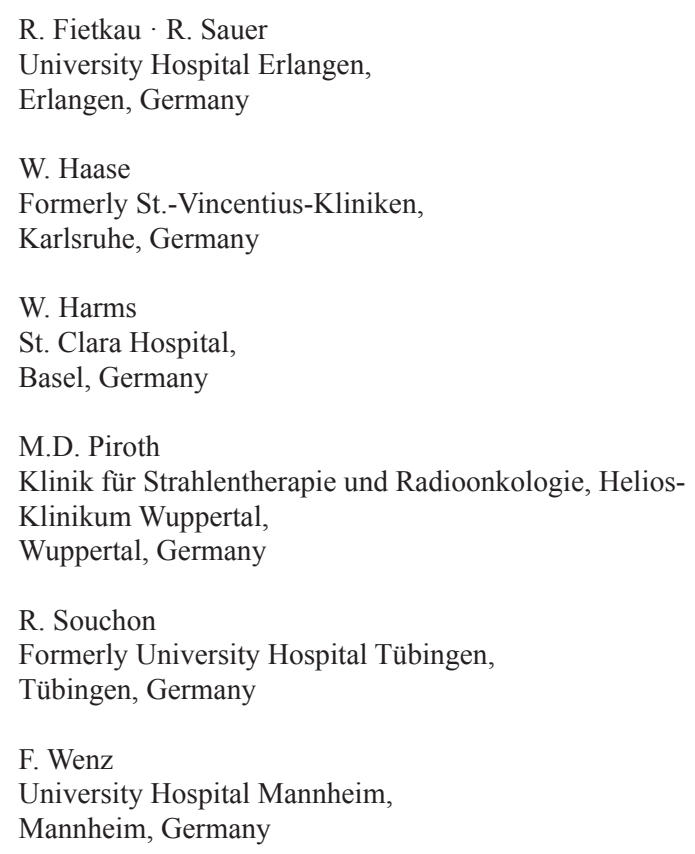


"no further local treatment". Adjuvant whole breast irradiation (WBI) with standard tangents was mandatory, third field nodal irradiation was explicitly excluded. No further requirements were made concerning RT technique or quality control. Systemic treatment was left to the discretion of the treating center. The study was terminated after accrual of 891 instead of the originally planned 1900 patients. After a median follow-up of 6.3 years, no difference in OAS and DFS was observed, 5-year locoregional recurrence-free survival rate was $96.7 \%$ after SNB alone and $95.7 \%$ in patients with ALND. The authors concluded that ALND might no longer be justified in T1-2 tumors even in case of 1-2 positive sentinel nodes.

Speculation evolved, to what extent inadvertent radiotherapy of the axilla had contributed to the low rate of locoregional recurrence. Several studies investigated the dose delivered to the axillary nodes by conventional tangential fields, and explored the possibility of achieving an improved coverage of level I by minor field extensions in cranial direction $[1,11,15]$. Haffty et al. proposed the use of "high tangents" in order to reduce the potentially increased risk of local recurrence for SN-positive patients [4]. This seemed a tempting compromise between RT of the complete axilla and total omission of any local treatment.

In order to quantify the extent of axillary radiation in the Z0011 trial, Jagsi et al. [5] took the attempt to analyze the radiation field design of the $\mathrm{Z} 0011$ study population by requesting the treatment records from the involved radiation oncologists for a central review. Completed case report forms were obtained for 605/856 patients, of those 540 $(89 \%)$ had received WBI. Additional treatment to the supraclavicular region was recorded in $89(15 \%)$ of these patients in this subgroup. Noteworthy, detailed RT records were only available for 228 patients, of whom 185 (81.1\%) received tangents alone. Information about the cranial field border was provided by 142 records. "High tangents" (defined as cranial tangent border $\leq 2 \mathrm{~cm}$ from the humeral head) were used in $50 \%$ of the ALND patients and $52.6 \%$ of the SLND group. Of the 228 patients with records reviewed, 43 $(18.9 \%)$ received directed regional nodal RT using $\geq$ three fields: 22 in the ALND arm and 21 in the SLND arm. Those receiving directed nodal $\mathrm{RT}$ had greater nodal involvement $(p<0.01)$ than those who did not. Overall, there was no significant difference between both treatment arms in the use of protocol-prohibited nodal fields.

The authors (one of whom is the first author of Z0011) commented these finding as "unexpected" [5]. This appears as a benevolent interpretation of a study which is commonly cited as main evidence for a change of practice and turns out to be performed with a substantial amount of previously unrecognized protocol violations.

We agree that in spite of these violations, the non- inferiority of SNB compared to ALND is corroborated, none- theless without supporting the assumption that positive SN have no impact on prognosis [2]. The reverse has been recently indicated by the results of a subgroup analysis of the NSABP-B 32 trial which was originally also designed to evaluate whether SLND alone was equivalent to complete ALND, albeit in primarily SN-negative women. Tissue blocks of SN obtained from patients with pathologically negative SN were centrally re-evaluated and occult metastases were detected in $15.9 \%$. Follow-up showed a small but significantly worse outcome of those patients with occult metastases compared to those who remained negative. The absolute difference in 5-y OAS was $1.2 \%$ (p0.03), DFS even $2.8 \%$ (p0.02) [16]. Several large retrospective cohort studies showed a similar trend even for microscopically positive nodes $[6,9]$.

Jagsi et al state that their observations "should not be taken to suggest that nodal RT administered to patients in the Z 0011 study was necessary or beneficial". They substantiate this statement by stressing that a subgroup had not been irradiated at all (about $11 \%$ in both arms). Should the reader take this further protocol violation as evidence that local treatment is dispensable for SN positive patients? In fact, the analysis demonstrated that the vast majority of the women did receive local treatment - as relevant radiation doses were delivered to the axilla. The ability of radiation to sterilize axillary metastases has recently been demonstrated by the EORTC 10981-22023 AMAROS study comparing ALND vs. dedicated axillary radiotherapy in pathologically positive SN. The 5-year axillary recurrence rate was $0.54 \%$ after ALND and $1.03 \%$ after RT. As the rate of lymphedema was significantly lower, the authors suggested RT as a standard instead of ALND in this situation [12].

In this context, it seems worth mentioning, that two large randomized trials recently provided evidence indicating that regional nodal irradiation (RNI) not only improves local control but also reduces metastases and prolongs survival. The EORTC 22922-10925 [10] study included 4.004 women stage with mostly pT $1-2$ tumors $(95 \%)$ and either involved axillary LN and/or a medially located primary tumor. Patients were randomized to receive postoperative RT either with or without RNI. After 10 years, patients with RNI had a small but significant improvement of $1.6 \%$ in OAS and $3 \%$ in disease-free as well as metastases-free survival (MFS). The positive impact of RNI on MFS has also been indicated by the NCIC-CTG MA.20 trial [17]. The study included 1.832 women with mostly $1-3$ positive axillary nodes $(85 \%)$ and $10 \%$ with negative nodes. Patients were randomized after breast conserving surgery and ALND to either WBI or WBI and additional RNI. The 5-year disease free survival (DFS) was significantly improved by $5.4 \%$ in the RNI-group $(p=0.003)$. This difference is twice as high as the absolute benefit in terms of 
local control $(2.3 \%)$ and therefore hypothetically attributable to the prevention of distant metastases.

In summary, the question how to best manage $\mathrm{SN}$ positive patients without ALND remains unresolved, even if omission of ALND is justifiable. As the authors finally emphasize, their findings must not be extrapolated to patients without any axillary radiation dose coverage, for instance, in case $\mathrm{n}$ prone breast treatment or partial breast irradiation. One important conclusion should be to require dose volume histograms routinely for WBI treatment plans [13]. Jagsi et al. [5] deserve merit for their attempt to quantify the radiation dose of the axilla in order to estimate the potential contribution of inadvertent RT to the outcome of Z0011. Unfortunately, the results of their survey rather obscure than elucidate the issue.

The paper is in so far extraordinary as it provides remarkable insights into the pitfalls of interpreting "evidence" and illustrates that the label "randomized study" should not mislead to draw premature conclusions. It is reassuring that in the last sentence of the paper the authors conclude "that given the findings of our study, it is not unreasonable to also consider additional nodal treatment in selected patients".

At least, a definitely agreeable statement!

\section{Compliance with ethical guidelines}

Conflict of interest M.-L. Sautter-Bihl, F. Sedlmayer, W. Budach, J. Dunst, P. Feyer, R. Fietkau, 'W. Haase, W. Harms, M.D. Piroth, R. Souchon, F. Wenz, R. Sauer declare no conflict of interest.

\section{References}

1. Alço G, Iğdem S, Ercan T et al (2010) Coverage of axillary lymph nodes with high tangentlial fields in breast radiotherapy. Br J Radiol 83:1072-1076

2. Engel J, Emeny RT, Hölzel D (2012) Positive lymph nodes do not metastasize. Cancer Metastasis Rev 31(1-2):235-246

3. Giuliano AE, Hunt KK, Ballman KV et al (2011) Axillary dissection vs. no axillary dissection in women with invasive breast cancer and sentinel node metastasis: a randomized clinical trial. JAMA 305:569-575
4. Haffty BG, Hunt KK, Harris JR et al (2011) Positive sentinel nodes without axillary dissection: implications for the radiation oncologist. J Clin Oncol 29:4479-4481

5. Jagsi R, Chadha M, Moni J et al (2014) Radiation field design in the ACOSOG Z0011 (Alliance). Trial J Clin Oncol 32

6. Lupe K, Truong PT, Alexander C et al (2011) Ten-Year locoregional recurrence risks in women with nodal micrometastatic breast cancer staged with axillary dissection. Int J Radiat Oncol Biol Phys 81:e681-e688

7. Murthy V, Ballehaninna UK, Chamberlain RS (2014) Unanswered questions about the role of axillary dissection in women with invasive breast cancer and sentinel node metastasis. Clin Breast Cancer 12:305-307

8. NCCN. Clinical practice guidelines in oncology: breast cancer, version 1.2014. National Comprehensive Cancer Network, www. ncen.org

9. Pepels MJ, de Boer M, Bult P et al (2012) Regional recurrence in breast cancer patients with sentinel node micrometastases and isolated tumor cells. Ann Surg 255:116-121

10. Poortmans P, Kirkove C, Budach V et al (2013) Irradiation of the internal mammary and medial supraclavicular lymph nodes in stage I to III breast cancer: 10 years results of the EORTC Radiation Oncology and Breast Cancer Groups phase III trial 22922/10925. EJC 49(Suppl 3)1 (abstract BA2)

11. Reznik J, Cicchetti MG, Degaspe B et al (2005) Analysis of axillary coverage during tangential radiation therapy to the breast. Int J Radiat Oncol Biol Phys 61:163-168

12. Rutgers EJ, Donker M, Straver ME et al (2013) Radiotherapy or surgery of the axilla after a positive sentinel node in breast cancer patients: final analysis of the EORTC AMAROS trial (10981/22023. J Clin Oncol 31 (Abstract LBA1001)

13. Sautter-Bihl ML, Sedlmayer F, Budach W et al (2014) Breast Cancer Expert Panel of the German Society of Radiation Oncology (DEGRO). DEGRO practical guidelines: radiotherapy of breast cancer III-radiotherapy of the lymphatic pathways. Strahlenther Onkol 190(4):342-351

14. S3. Interdisziplinäre S3-Leitlinie für die Diagnostik, Therapie und Nachsorge des Mammakarzinoms, Langversion 3.0, Aktualisierung 2012, AWMF-Register-Nummer: 032-045OL 2012

15. Schlembach PJ, Buchholz TA, Ross MI et al (2001) Relationship of sentinel and axillary level I-II lymph nodes to tangential fields used in breast irradiation. Int J Radiat Oncol Biol Phys 51:671-678

16. Weaver DL, Ashikaga T, Krag DN et al (2011) Effect of occult metastases on survival in node-negative breast cancer. N Engl J Med 364:412-421

17. Whelan T, Olivotto I, Ackerman I et al (2011) NCIC-CTG MA. 20: an intergroup trial of regional nodal irradiation in early breast cancer. J Clin Oncol 29(suppl) (abstract LBA 1003) 